\title{
Adaptive and Robust Methods of Reconstruction (ARMOR) for Thermoacoustic Tomography
}

\author{
Yao Xie*, Student Member, IEEE, Bin Guo, Member, IEEE, Jian Li, Fellow, IEEE, \\ Geng $\mathrm{Ku}$, and Lihong V. Wang, Fellow, IEEE
}

\begin{abstract}
In this paper, we present new adaptive and robust methods of reconstruction (ARMOR) for thermoacoustic tomography (TAT), and study their performances for breast cancer detection. TAT is an emerging medical imaging technique that combines the merits of high contrast due to electromagnetic or laser stimulation and high resolution offered by thermal acoustic imaging. The current image reconstruction methods used for TAT, such as the delay-and-sum (DAS) approach, are data-independent and suffer from low-resolution, high sidelobe levels, and poor interference rejection capabilities. The data-adaptive ARMOR can have much better resolution and much better interference rejection capabilities than their data-independent counterparts. By allowing certain uncertainties, ARMOR can be used to mitigate the amplitude and phase distortion problems encountered in TAT. The excellent performance of ARMOR is demonstrated using both simulated and experimentally measured data.
\end{abstract}

Index Terms-Array signal processing, biomedical acoustic imaging, robustness.

\section{INTRODUCTION}

$\mathbf{T}$ HERMOACOUSTIC tomography (TAT), the earliest investigation of which dates back to the 1980s [1], has recently attracted much interest with its great promise in a wide span of biomedical applications (see, e.g., [2]-[4]). Its physical basis lies in the contrast of the radiation absorption rate among different biological tissues. Due to the thermoacoustic effect, when a short electromagnetic pulse (e.g., microwave or laser) is absorbed by the tissue, the heating results in expansion that generates acoustic signals. In TAT, an image of the tissue absorption properties is reconstructed from the recorded thermoacoustic signals. Such an image may reveal the physiological and pathological status of the tissue, which can be useful in many

Manuscript received May 8, 2007; revised October 22, 2007. First published February 25, 2008; current version published December 17, 2008. This work was supported in part by the National Institutes of Health (NIH) under Grant 1R41CA107903-1, in part by the U.S. Army Medical Command under Contract W81XWH-06-1-0389, and in part by the National Natural Science Foundation of China under Grant 60428101. Asterisk indicates corresponding author.

*Y. Xie was with the Department of Electrical and Computer Engineering, University of Florida, Gainesville, FL 32611-6130 USA. She is now with the Department of Electrical Engineering, Stanford University, Stanford, CA 94305 USA.

B. Guo was with the Department of Electrical and Computer Engineering, University of Florida, Gainesville, FL 32611 USA. He is now with the Department of Electrical and Computer Engineering, Duke University, Durham, NC 27708 USA.

$\mathrm{J}$. Li is with the Department of Electrical and Computer Engineering, University of Florida, Gainesville, FL 32611 USA.

G. Ku and L. V. Wang are with the Department of Biomedical Engineering, School of Engineering and Applied Science, Washington University in St. Louis, St. Louis, MO 63130-4899 USA.

Color versions of one or more of the figures in this paper are available online at http://ieeexplore.ieee.org.

Digital Object Identifier 10.1109/TBME.2008.919112 applications including breast cancer detection [5]. Compared with microwave imaging and ultrasound imaging, TAT combines their merits and possesses both fine imaging resolution and good spatial contrast properties [4].

Developing accurate and robust image reconstruction methods is one of the key challenges encountered in TAT. Various image reconstruction algorithms have been developed for TAT. By using Radon transformation on the TAT data function, reflectivity tomography reconstruction algorithms can be used for TAT image reconstruction [6]. Exact inverse solutions have been found for different scanning geometries in both the frequency domain [7], [8] and the time domain [9], [10]. Approximate reconstruction algorithms, such as the time-domain delay-andsum (DAS) beamforming method [11], [12] and the optimal statistical approach [13], have also been proposed. However, a common assumption of these existing methods is that the surrounding tissue is acoustically homogeneous. This approximation is inadequate in many medical imaging applications. According to previous studies, the sound speed in human female breast varies widely from 1430 to $1570 \mathrm{~m} / \mathrm{s}$ around the commonly assumed speed of $1510 \mathrm{~m} / \mathrm{s}$ [14], [15]. The heterogeneous acoustic properties of biological tissues cause amplitude and phase distortions in the recorded acoustic signals, which can result in significant degradation in imaging quality [16].

In ultrasound tomography (UT), wavefront distortion due to heterogeneity of biological tissue has been studied extensively. Various wavefront correction methods have been proposed [17]. However, they are not highly effective at correcting severe amplitude distortions [18], and they usually involve complicated procedures. The problem in TAT is somewhat different from that in UT. In the breast UT, the amplitude distortion caused by refraction is more problematic than the phase distortion induced by acoustic speed variation. In TAT, however, even for the biological tissue, such as the breast tissue, with a relatively weak heterogeneity, phase distortion dominates amplitude distortion [16]. These unique features suggest that new adaptive and robust imaging techniques should be designed especially for TAT.

Time-domain approximate reconstruction algorithms, such as the DAS (weighted or unweighted) type of data-independent approaches have various applications in medical imaging. They need little prior information on the tissue for image reconstruction and can be fast and simple to implement to process the wideband acoustic signals. Although not based on the exact solution, they provide similar image qualities to those of the exact reconstruction algorithms. However, these data-independent methods tend to suffer from poor resolution and high-sidelobelevel problems. Data-adaptive approaches, such as the recently 
introduced robust Capon beamforming (RCB) method [19], can have much better resolution and much better interference rejection capability than their data-independent counterparts.

We propose adaptive and robust methods of reconstruction (ARMOR) based on RCB for TAT. ARMOR can be used to mitigate the amplitude and phase distortion problems in TAT by allowing certain uncertainties. Specifically, in the first step of ARMOR, RCB is used for waveform estimation by treating the amplitude distortion with an uncertainty parameter. In the second step of ARMOR, a simple, yet effective, peak searching method is used for phase distortion correction. Compared with other energy- or amplitude-based response intensity estimation methods, peak searching can be used to improve image quality with little additional computational costs. Moreover, since the acoustic pulse is usually bipolar: a positive peak, corresponding to the compression pulse, and a negative peak, corresponding to the rarefaction pulse [11], we can further enhance the image contrast in TAT by using the peak-to-peak difference as the response intensity for a focal point. We will demonstrate the excellent performance of ARMOR by using both data simulated on a 2-D breast model and data experimentally measured from mastectomy specimens.

The remainder of this paper is organized as follows. In Section II, we formulate the problem of interest. Sections III-V describe the first, second, and third steps of ARMOR, respectively. Examples based on simulated and real-world experimental data are presented in Section VI. Finally, Section VII provides the conclusions.

\section{Problem Formulation}

Consider a TAT imaging system, as shown in Fig. 1(a). A stimulating electromagnetic (laser or microwave) pulse is absorbed by the biological tissue under testing, which causes a sudden heat change (of the order of $10^{-4}{ }^{\circ} \mathrm{C}$ [20]). Due to the thermoacoustic effect, an acoustic pulse is generated that can be recorded by an ultrasonic transducer array. The transducer array may be a real aperture array or a synthetic aperture array formed by rotating a sensor around the tissue and recording the acoustic waves at different locations. We assume that the number of transducers in the array (or in the synthetic aperture array case, the number of transducer data acquisition locations) is $M$. Each transducer is assumed to be omnidirectional; mutual couplings among the transducers are not considered in our model as they can be tolerated by our robust algorithms to a certain extent. The recorded acoustic signals are sufficiently sampled and digitized and a typical recorded pulse is shown in Fig. 1(b) (based on the data measured on the breast specimen II described in Section VI).

The data model for the sampled and digitized acoustic signal recorded by the $m$ th transducer is given by:

$$
x_{m}(n)=s_{m}(n)+\tilde{e}_{m}(n), \quad m=1, \ldots, M .
$$

where $n$ is the discrete time index, starting from $t_{0}$ after the excitation pulse. The scalar $s_{m}(n)$ denotes the signal component, which corresponds to the acoustic pulse generated at a focal point, and $\tilde{e}_{m}(n)$ is the residual term, which includes unmod-

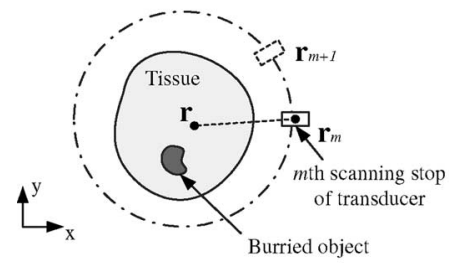

(a)

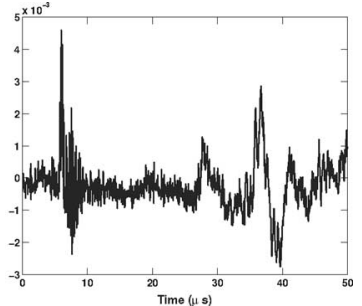

(b)
Fig. 1. (a) A schematic of a 2-D synthetic-aperture-based TAT scanning system. (b) A typical acoustic pulse recorded by a transducer (for data measured from breast specimen II)

eled noise and interference (caused by other sources within the tissue).

The goal of ARMOR is to reconstruct an image of thermoacoustic response intensity $I(\mathbf{r})$, which is directly related to the absorption property of the tissue, from the recorded data set $\left\{x_{m}(n)\right\}$. Herein, the (2-D or 3-D) vector $\mathbf{r}$ denotes the focal point location coordinate. To form an image, we scan the focal point location $\mathbf{r}$ to cover the entire cross section of the tissue (the transducers can acquire signals at different heights; for each height, a 2-D cross-sectional image can be reconstructed and a 3 -D image can be formed from the 2-D images). We allow certain uncertainties in ARMOR to deal with amplitude and phase distortions caused by the background heterogeneity.

The discrete arrival time of the pulse (for the $m$ th transducer) can be determined approximately as

$$
t_{m}(\mathbf{r})=\left\lfloor-\frac{t_{0}}{\Delta t}+\frac{\left\|\mathbf{r}-\mathbf{r}_{m}\right\|}{\Delta t v_{0}}\right\rfloor .
$$

We will omit the dependence of the arrival time $t_{m}(\mathbf{r})$ on $\mathbf{r}$ hereafter for notational simplicity. Here, $\Delta t$ is the sampling interval, and the 3-D vector $\mathbf{r}_{m}$ denotes the location of the $m$ th transducer. The sound speed $v_{0}$ is chosen to be the average sound speed of the biological tissue under interrogation. The notation $\|\mathbf{x}\|$ denotes the Euclidean norm of $\mathbf{x}$, and $\lfloor y\rfloor$ stands for rounding to the greatest integer less than $y$. The second term in (2) represents the time-of-flight between the focal point and the $m$ th transducer.

The signal components $\left\{s_{m}(n)\right\}_{m=1}^{M}$ are approximately scaled and shifted versions of a nominal waveform $s(t)$ at the source

$$
s_{m}(n) \approx \frac{\exp \left(-\alpha\left\|\mathbf{r}-\mathbf{r}_{m}\right\|\right)}{\left\|\mathbf{r}-\mathbf{r}_{m}\right\|} \cdot s\left(n-t_{m}\right)
$$

where $\alpha$ is the attenuation coefficient in Nepers $/ \mathrm{m}$. In TAT, the major frequency components of the acoustic signals take a relatively narrow band, and are usually lower than those in UT [16]. Hence, we can approximate $\alpha$ as a frequency-independent constant.

We preprocess the data to time delay all the signals from the focal point $\mathbf{r}$ and compensate for the loss in amplitude due to propagation decay. Let $y_{m}(n)$ denote the signal after preprocessing to backpropagate the detected signal to the source

$$
y_{m}(n)=\exp \left(\alpha\left\|\mathbf{r}-\mathbf{r}_{m}\right\|\right) \cdot\left\|\mathbf{r}-\mathbf{r}_{m}\right\| \cdot x_{m}\left(n+t_{m}\right) .
$$


Then, the received vector data model can be written as

$$
\mathbf{y}(n)=\mathbf{a}_{0} s(n)+\mathbf{e}(n), \quad n=-N, \cdots, N
$$

where $\mathbf{a}_{0}$ is the corresponding steering vector, which is approximately equal to $\overline{\mathbf{a}}=[1, \ldots, 1]^{T}, \mathbf{y}(n)=\left[y_{1}(n), \ldots, y_{M}(n)\right]^{T}$, $\mathbf{e}(n)$ represents the noise and interference term after preprocessing, and $(\cdot)^{T}$ denotes the transpose. Here, we define the time interval of interests for the signal $\mathbf{y}(t)$ to be from $-N$ to $N$, which means that we only take $N$ samples before and after the approximate arrival time given in (2) for the focal point at $\mathbf{r}$. The value of $N$ should be chosen large enough so that the interval from $-N$ to $N$ covers the expected signal duration in the region of interest.

In reality, both the amplitude and the phase (or pulse arrival time) of the acoustic pulse will be distorted. A major cause for these distortions is the acoustically heterogeneous background. Amplitude distortion is mainly due to the interferences caused by multipath, which is inevitable in the heterogeneous medium: refraction occurs due to acoustic speed mismatch across the tissue interface; consequently, acoustic pulses arrived at the transducer will be via different routes and interfere with each other. On the other hand, phase distortion is mainly caused by the nonuniform sound speed. For example, in human female breast, the sound speed can vary from 1430 to $1570 \mathrm{~m} / \mathrm{s}$; therefore, the actual arrival time will fluctuate around the approximately calculated time given in (2). Moreover, an inaccurate estimate of $t_{0}$ ( $t_{0}$ is aligned with the focal point's signal arrival time) and the transducer calibration error may also contribute to the phase distortion. Amplitude and phase distortion will blur the image, raise the image background noise level, lower the values of the object of interest, and, consequently, decrease the image contrast [16].

We mitigate the effects of these distortions by allowing $\mathbf{a}_{0}$ to belong to an uncertainty set centered at $\overline{\mathbf{a}}$ and by considering the signal arriving within the interval from $-N$ to $N$.

\section{STEP I OF ARMOR: WAVEFORM ESTIMATION}

The first step of ARMOR is to estimate the waveform of the acoustic pulse generated by the focal point at location $\mathbf{r}$, based on the data model in (5). It will appear that we have neglected the presence of phase distortion by using this data model in the first step. However, by allowing $\mathbf{a}_{0}$ to be uncertain, we can tolerate some phase distortions as well. This approximation causes little performance degradation to our robust algorithm.

Covariance-fitting-based RCB [21] is used to first estimate the steering vector $\mathbf{a}_{0}$, and use the estimated $\mathbf{a}_{0}$ to obtain an optimal beamformer weight vector for pulse waveform estimation. By assuming that the true steering vector lies in the vicinity of the nominal steering vector $\overline{\mathbf{a}}$, we consider the following optimization problem [19]

$$
\begin{array}{ll}
\max _{\sigma^{2}, \mathbf{a}_{0}} \sigma^{2} \quad \text { subject to } \quad & \hat{\mathbf{R}}-\sigma^{2} \mathbf{a}_{0} \mathbf{a}_{0}^{T} \succeq 0 \\
& \left\|\mathbf{a}_{0}-\overline{\mathbf{a}}\right\|^{2} \leq \varepsilon
\end{array}
$$

where $\mathbf{A} \succeq 0$ means that the matrix $\mathbf{A}$ is positive semidefinite, $\sigma^{2}$ is the power of the signal of interest, and

$$
\hat{\mathbf{R}}=\frac{1}{2 N+1} \sum_{n=-N}^{N} \mathbf{y}(n) \mathbf{y}^{T}(n)
$$

is the sample covariance matrix. The second constraint in (6) is a spherical uncertainty set; an elliptical uncertainty set can be used instead, if a tighter constraint is desirable [21].

The parameter $\varepsilon$ in (6) determines the size of the uncertainty set and is a user parameter. To avoid the trivial solution of $\mathbf{a}_{0}=0$, we require that

$$
\varepsilon<\|\overline{\mathbf{a}}\|^{2} .
$$

It can be verified that the smaller the $\varepsilon$, the higher the resolution and the stronger the ability of RCB to suppress an interference that is close to the signal of interest, and that the larger the $\varepsilon$, the more robust RCB will be to tolerate distortions and smallsample-size problems caused by calculating $\hat{\mathbf{R}}$ in (7) from a finite number of data vectors or snapshots. When $\varepsilon$ is close to $M, \mathrm{RCB}$ will perform like DAS. To attain high resolution and to effectively suppress interference, $\varepsilon$ should be made as small as possible. On the other hand, the smaller the sample size $N$ or the larger the distortions, the larger should $\varepsilon$ be chosen [19]. Since the performance of RCB does not depend very critically on the choice of $\varepsilon$ (as long as it is set to be a "reasonable value") [21], such qualitative guidelines are usually sufficient for making a choice of $\varepsilon$. We will investigate the effect of $\varepsilon$ in Section VI. In our examples in Section VI, we choose certain reasonable initial values for $\varepsilon$, and then make some adjustments empirically based on image quality: making it smaller when the resulting images have low resolution, or making it larger when the image is distorted by interferences.

By using the Lagrange multiplier method, the solution to (6) is given by [19]

$$
\hat{\mathbf{a}}_{0}=\overline{\mathbf{a}}-[\mathbf{I}+\mu \hat{\mathbf{R}}]^{-1} \overline{\mathbf{a}}
$$

where $\mathbf{I}$ is the identity matrix, and $\mu \geq 0$ is the corresponding Lagrange multiplier that can be solved from the following equation

$$
\left\|(\mathbf{I}+\mu \hat{\mathbf{R}})^{-1} \overline{\mathbf{a}}\right\|^{2}=\varepsilon .
$$

Consider the eigendecomposition on the sample covariance matrix $\hat{\mathbf{R}}$

$$
\hat{\mathbf{R}}=\mathbf{U} \boldsymbol{\Gamma} \mathbf{U}^{T}
$$

where the columns of $\mathbf{U}$ are the eigenvectors of $\hat{\mathbf{R}}$ and the diagonal matrix $\Gamma$ consists of the corresponding eigenvalues $\gamma_{1} \geq \gamma_{2} \geq \cdots \geq \gamma_{M}$. Let $\mathbf{b}=\mathbf{U}^{T} \overline{\mathbf{a}}$, where $b_{m}$ denotes its $m$ th element. Then, (10) can be rewritten as

$$
\mathcal{L}(\mu)=\sum_{m=1}^{M} \frac{\left|b_{m}\right|^{2}}{\left(1+\mu \gamma_{m}\right)^{2}}=\varepsilon .
$$

Note that $\mathcal{L}(\mu)$ is a monotonically decreasing function of $\mu$, with $\mathcal{L}(0)>\varepsilon$ by $(8)$ and $\lim _{\mu \rightarrow \infty} \mathcal{L}(\mu)=0<\varepsilon$, which means that $\mu$ can be solved efficiently, say, by using the Newton's method 
(see [19] for more details). After obtaining the value of $\mu$, the estimate $\hat{\mathbf{a}}_{0}$ of the actual steering vector $\mathbf{a}_{0}$ is determined by (9).

Observe that there is a "scaling ambiguity" in (6) by treating both the signal power $\sigma^{2}$ and the steering vector $\mathbf{a}_{0}$ as unknowns (see [19] and [21]). The ambiguity exists in the sense that $\left(\sigma^{2}, \mathbf{a}_{0}\right)$ and $\left(\sigma^{2} / c, c^{1 / 2} \mathbf{a}_{0}\right)$ (for any constant $c>0$ ) yield the same term $\sigma^{2} \mathbf{a}_{0} \mathbf{a}_{0}^{T}$. To eliminate this ambiguity, we scale the solution $\hat{\mathbf{a}}_{0}$ to make its norm satisfy the following condition

$$
\left\|\hat{\mathbf{a}}_{0}\right\|^{2}=M \text {. }
$$

(Note that $M=\|\overline{\mathbf{a}}\|^{2}$.)

To obtain an estimate for the signal waveform $s(n)$, we apply a weight vector to the preprocessed signals $\{\mathbf{y}(n)\}_{n=-N}^{N}$. The weight vector is determined by using the estimated steering vector $\hat{\mathbf{a}}_{0}$ in the weight vector expression of the standard Capon beamformer (see, e.g., [19] and [21])

$$
\hat{\mathbf{w}}_{\mathrm{RCB}}=\frac{\left\|\hat{\mathbf{a}}_{0}\right\|}{M^{1 / 2}} \cdot \frac{\left[\hat{\mathbf{R}}+\frac{1}{\mu} \mathbf{I}\right]^{-1} \overline{\mathbf{a}}_{0}}{\overline{\mathbf{a}}_{0}^{T}\left[\hat{\mathbf{R}}+\frac{1}{\mu} \mathbf{I}\right]^{-1} \hat{\mathbf{R}}\left[\hat{\mathbf{R}}+\frac{1}{\mu} \mathbf{I}\right]^{-1} \overline{\mathbf{a}}_{0}} .
$$

Note that (14) has a diagonal loading form, which allows the sample covariance matrix to be rank-deficient. The beamformer output can be written as

$$
\hat{s}_{\mathrm{RCB}}(n)=\hat{\mathbf{w}}_{\mathrm{RCB}}^{T} \mathbf{y}(n), \quad n=-N, \ldots, N
$$

which is the waveform estimate for the acoustic pulse generated at the focal point at location $\mathbf{r}$.

RCB can provide a much better waveform estimate than the conventional DAS but at a higher computational cost. For a single focal point, RCB requires $O\left(M^{3}\right)$ flops, which mainly come from the eigendecomposition of the sample covariance matrix $\hat{\mathbf{R}}$ [19]; DAS needs only $O(M)$ flops. DAS can be used as a fast image reconstruction method to provide initial imaging results.

The weight vector used by DAS for waveform estimation is

$$
\hat{\mathbf{w}}_{\mathrm{DAS}}=\overline{\mathbf{a}}
$$

and the estimated waveform is given by

$$
\hat{s}_{\mathrm{DAS}}(n)=\hat{\mathbf{w}}_{\mathrm{DAS}}^{T} \mathbf{y}(n)=\sum_{m=1}^{M} y_{m}(n), \quad n=-N, \ldots, N .
$$

\section{STEP II OF ARMOR: PEAK SEARCHING}

Based on the estimated waveform obtained in Step I for the focal point at location $\mathbf{r}$, in Step II of ARMOR, we will search for the two peaks of the bipolar acoustic pulse generated by the focal point. In a homogeneous background, where phase distortion is absent, we can accurately calculate the arrival time of the acoustic pulse generated by the focal point at location $\mathbf{r}$ by using (2). However, this is never true in heterogeneous biological tissues. It was reported in [16] that when the heterogeneity is weak, such as in the breast tissue, amplitude distortion caused by multipath is not severe. We can assume that the original peak remains a peak in the waveform estimated from Step I of ARMOR.
The bipolar acoustic pulse has one peak positive and another negative. We determine the positive and negative peak values as follows:

$$
\begin{aligned}
& P^{+}=\max \left\{\max _{n \in[-\Delta, \Delta]} \hat{s}(n) 0\right\}, \\
& P^{-}=\min \left\{\min _{n \in[-\Delta, \Delta]} \hat{s}(n) 0\right\},
\end{aligned}
$$

where the searching range $[-\Delta, \Delta] \in[-N, N]$ is around the calculated arrival time given by (2). Here $\Delta$ is a user parameter. Since the peak searching is independent of the particular waveform estimation methods, we use $\hat{s}(n)$ to denote the waveform estimated by either DAS or ARMOR.

The search range is determined by the difference between the true arrival time $\bar{t}_{m}$ and the calculated arrival time $t_{m}$, based on (2). This arrival time difference has been analyzed for breast tissue by taking into account its relatively weak heterogeneity acoustic property [16]. An expression for this difference is given in [16] by

$$
\delta_{m}\left(\mathbf{r}^{\prime}\right)=\bar{t}_{m}-t_{m} \propto \frac{\left[v\left(\mathbf{r}^{\prime}\right)-v_{0}\right]}{v_{0}}
$$

where $\mathbf{r}^{\prime}$ is a point within the line connecting the focal point at location $\mathbf{r}$ and the $m$ th transducer at location $\mathbf{r}_{m}$, and $v\left(\mathbf{r}^{\prime}\right)$ is the local sound speed. The higher order terms of $\left[v\left(\mathbf{r}^{\prime}\right)-\right.$ $\left.v_{0}\right] / v_{0}$ in (20) have been ignored. It is reasonable to assume that $v\left(\mathbf{r}^{\prime}\right)$ is Gaussian-distributed with mean $v_{0}$ and variance $\sigma_{v}^{2}$. Consequently, the arrival time difference is also Gaussiandistributed with zero-mean and variance $\sigma_{\delta}^{2} \propto \sigma_{v}^{2} / v_{0}^{2}$. If we choose $\Delta=\sigma_{\delta}$, and the duration of the acoustic pulse is $\tau$, we can find the two peaks of the pulse within the interval $\left(-\sigma_{\delta}, \sigma_{\delta}+\right.$ $\tau)$ on the recorded signals with a high probability of 0.6826 . This analysis is consistent with the experimental measurements in [22]. From our examples, we found that a symmetric range $[-\Delta, \Delta]$ around the estimated arrival time performs similarly to the asymmetric range $[-\Delta, \Delta+\tau]$, and we use the former since it is easy to handle in practice. Also, we can use similar techniques as those in [22] to estimate $\sigma_{\delta}$ to find a good searching range for Step II of ARMOR, and to estimate $\tau$ for the energytype methods, as shown in our examples later.

There is a tradeoff in choosing the searching range. The larger the searching range, the higher the probability we can find the peaks of the acoustic pulse within the range. However, if the range is chosen too large, the interferences may cause false peaks, and as a consequence, we are more likely to find a false peak. In our examples in Section VI, we choose the best searching range empirically based on the estimated variance of the arrival time difference $\hat{\sigma}_{\delta}$.

\section{STEP III OF ARMOR: INTENSITY CALCULATION}

After estimating the waveform generated by the focal point at location $\mathbf{r}$, we need to obtain the response intensity based on the estimated waveform. For the same estimated waveform, different approaches can be used to evaluate the focal point response intensity. These approaches extract different information from 
the estimated waveform as the response intensity, and may be useful to physicians in different ways.

There are two major types of response intensity measurement approaches: amplitude-based and energy-based. The waveform peak values obtained in Step II of ARMOR can be used for both approaches.

Conventional DAS uses the amplitude-based measure for TAT imaging [11], [12], with the corresponding response intensity given by $\hat{s}(0)$, or equivalently

$$
I_{\mathrm{C}}=\hat{s}(0)=\sum_{m=1}^{M} y_{m}(0)
$$

where the subscript "C" stands for "Conventional."

The energy-based measure, such as the one used in [23], calculates the response intensity as follows

$$
I_{\mathrm{E} 1}=\hat{s}^{2}(0)=\left[\sum_{m=1}^{M} y_{m}(0)\right]^{2}
$$

where the subscript "E1" means "Energy-type 1."

The entire pulse energy has also been used as an intensity measure, such as in the monostatic and multistatic microwave imaging for breast cancer detection [24], [25], and the intensity is given by

$$
I_{\mathrm{E} 2}=\sum_{n=0}^{\tau} \hat{s}^{2}(n)=\sum_{n=0}^{\tau}\left[\sum_{m=1}^{M} y_{m}(n)\right]^{2},
$$

where the subscript "E2" stands for "Energy-type 2."

We can consider using the peak value as the response intensity measure due to the bipolar nature of the response at the focal point

$$
I_{\mathrm{P}}= \begin{cases}P^{+}, & \text {if }\left|P^{+}\right| \geq\left|P^{-}\right| \\ P^{-}, & \text {otherwise }\end{cases}
$$

where the subscript " $\mathrm{P}$ " stands for "Peak," with $P^{+}$and $P^{-}$ defined in (18) and (19), respectively. Herein, we keep the sign of the maximum amplitude since the sign of the peak may also contain some information about the focal point.

Peak searching maximizes the output signal-to-noise ratio. An intuitive explanation is that, given the fact that the acoustic pulse is bipolar [11], if we assume that the residual term $\mathbf{e}(t)$ is stationary, or its power is uniform over time, then the signal-tonoise ratio (SNR) is maximized at the (positive or negative) peak of the acoustic pulse. As a comparison, the conventional DAS (21) fixes the samples to be summed up at the calculated arrival time. Due to phase distortions, the waveform at the calculated time may be far from the peak value.

We can also employ peak-to-peak difference as the response intensity for the focal point at location $\mathbf{r}$

$$
I_{\mathrm{PP}}=P^{+}-P^{-} \geq 0
$$

where the subscript "PP" denotes the "peak-to-peak difference." Peak-to-peak difference has higher imaging contrast than peak value measure: the peak-to-peak difference of the bipolar pulse is approximately twice the absolute peak value, which means that the output signal power of the former is four times of the

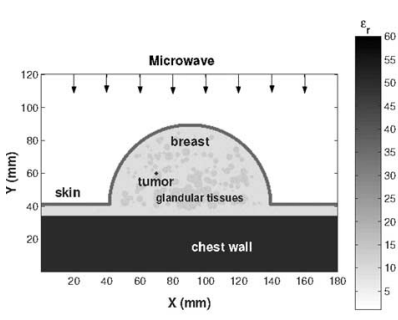

(a)

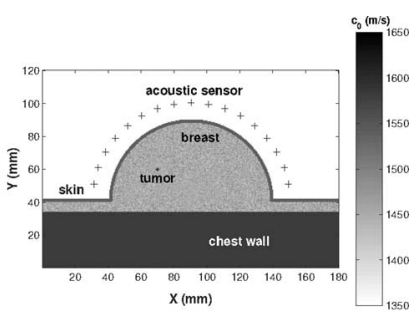

(b)
Fig. 2. 2-D breast model in an $x-y$ coordinate system, with a 2-mm-diameter tumor present. (a) Model for electromagnetic simulation. (b) Model for acoustic simulation.

latter; yet, the noise power of the former may be only twice that of the latter. Therefore, the output SNR may be doubled by using the peak-to-peak difference rather than the peak value. Both peak-value and peak-to-peak difference measures belong to the amplitude-based measures.

\section{NUMERICAL AND EXPERIMENTAL EXAMPLES}

We demonstrate the performance of ARMOR using both numerically simulated and experimentally measured TAT data. The ARMOR images are compared with the DAS images.

\section{A. Numerical Examples}

We consider a 2-D breast model, as shown in Fig. 2. The 2-D breast model includes 2-mm thick skin, chest wall, as well as randomly distributed fatty breast tissues and glandular tissues. The cross section of the breast model is a half-circle with a $10 \mathrm{~cm}$ diameter. In the first numerical example, a 2-mm-diameter tumor is located at $2.2 \mathrm{~cm}$ below the skin (at $x=7.0 \mathrm{~cm}, y=6.0 \mathrm{~cm}$ ). Fig. 2 shows the shape, dielectric properties, and sound speed variations of the breast model, as well as the tumor size and location for the first example. In the second numerical example, one large tumor ( $1 \mathrm{~cm}$ in diameter) is located at $x=12 \mathrm{~cm}$, $y=15 \mathrm{~cm}$. Other properties of the breast model for the second example are the same as those for the first example.

To reduce the reflections from the skin, the breast model is immersed in a lossless liquid with permittivity similar to that of the breast fatty tissue. Seventeen transducers (assumed omnidirectional) are located on a half-circle $10 \mathrm{~mm}$ away from the skin, with uniform spacing, to form a real aperture array.

The dielectric properties of the breast tissues are assumed to be Gaussian random variables with variations of $\pm 10 \%$ around their nominal values. This variation represents the upper bound reported in the literature. The nominal values are chosen to be typical of those reported in the literature [5], [26], which is given in Table I [24]. The dielectric constants of glandular tissues are between $\epsilon_{r}=11$ and $\epsilon_{r}=15$. The dispersive properties of the fatty breast tissue and those of the tumor are also considered in the model. The randomly distributed breast fatty tissues and glandular tissues with variable dielectric properties are representative of the nonhomogeneity of the breast of an actual patient. 
TABLE I

ACRONYMS

\begin{tabular}{|l|l|}
\hline ART & Adaptive and Robust Methods Of Reconstruction \\
\hline DAS & Delay-And-Sum \\
\hline FDTD & Finite Difference Time Domain \\
\hline PML & Perfectly matched layer \\
\hline RCB & Robust Capon Beamforming \\
\hline SNR & Signal-to-Noise Ratio \\
\hline SAR & Specific Absorption \\
\hline TAT & Thermoacoustic Tompgraphy \\
\hline UT & Ultra-sound Tomography \\
\hline C & Conventional \\
\hline E1 & Energy-type 1 \\
\hline E2 & Energy-type 2 \\
\hline P & Peak \\
\hline PP & Peak-to-Peak difference \\
\hline
\end{tabular}

Following the report that the breast tissues have a weak acoustic heterogeneity [16], we model the sound speed within the breast as a Gaussian random variable with variation $\pm 5 \%$ around the assumed average sound speed of $1500 \mathrm{~m} / \mathrm{s}$. Since the attenuation coefficient $\alpha$ in (3) is small for breast tissue $(0.75 \mathrm{~dB} /(\mathrm{MHz} \cdot \mathrm{cm}))[15]$ and the acoustic signals are below $2 \mathrm{MHz}$, we neglect the exponential attenuation in acoustic wave propagation. Also, since the acoustic pressure field generated by the thermoacoustic effect is usually small [20], we do not consider the nonlinear acoustic effects. The probing microwave pulse used here is a modulated rectangular pulse with a modulating frequency of $800 \mathrm{MHz}$. The duration of the pulse is $1 \mu \mathrm{s}$. More details about the thermal acoustic simulations are given in the Appendix. In the following, all the images are displayed on a linear scale, and we will name the imaging methods by their waveform estimation method followed by the intensity calculation approach, such as "DAS-C."

Note that the skin also absorbs microwave energy and generates acoustic signals. The skin response is much stronger than that of the tumor, since the skin has a much larger area than the tumor and the skin is closer to the acoustic sensors. So, before applying the aforementioned preprocessing steps and ARMOR, we remove the strong skin response using techniques similar to those in [24]. A calibration signal is obtained as the average of the recorded signals containing similar skin response. Then, the calibration signal is subtracted out from all recorded signals to remove the skin response as much as possible.

The searching range is chosen by the guidelines presented in Section IV. To obtain a general profile of the arrival time difference caused by the phase distortion, we use a simple method similar to the one used in [27]. First, the cross-correlation functions for all the signals recorded by the two adjacent transducers are obtained. The peak value of the cross-correlation function is used to estimate the arrival time delay between the signals recorded by the adjacent transducers. Second, these arrival time delays are fitted using a fourth-order polynomial curve, which is dominated by the arrival time delays due to the path length differences in the absent of phase distortions. The fourth-order polynomial is used since the delay caused by the path length difference should vary smoothly [27]. Fig. 3(a) shows the estimated arrival time delay and the delay based on curve fitting.

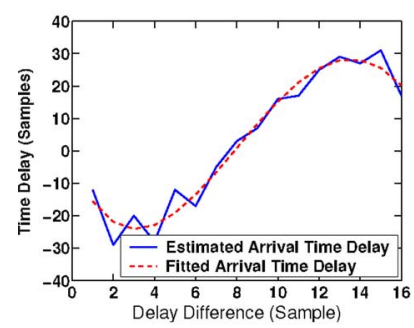

(a)

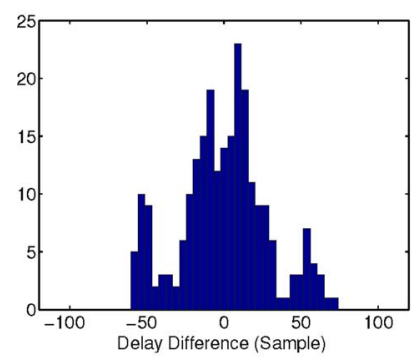

(c)

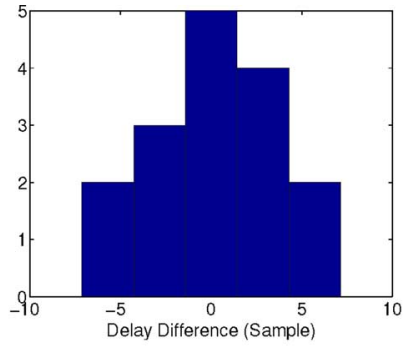

(b)

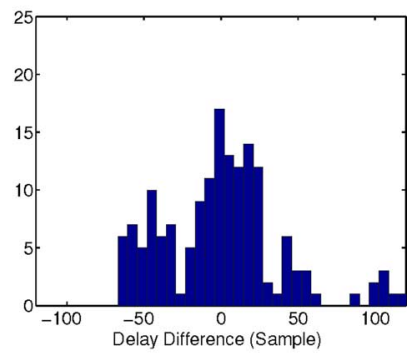

(d)
Fig. 3. (a) Comparison between the estimated and fitted arrival time delays, for the simulated breast model with one tumor (the curves for the two-tumor case are similar). Histograms of delay differences. (b) Simulated breast model with one tumor. (c) Breast specimen I. (d) Breast specimen II.

Third, the delay difference between the estimated arrival time delay and the fitted delay, or the fitting error, is treated as the arrival time distortion for the transducers. The standard deviation of the delay difference is used to estimate $\sigma_{\delta}$. Although the accuracy of the cross-correlation method is limited due to false peaks and jitter problems, it is sufficient to obtain a qualitative profile for $\sigma_{\delta}$.

Fig. 3 gives the histogram of the delay difference for all the cases that we considered herein. For the simulated example, the standard deviation of the delay difference is 4.5 , which indicates a weak phase distortion in the breast model. We set an initial value for $\Delta$, based on the estimated $\hat{\sigma}_{\delta}$, and adjust the length of the searching range to achieve the best imaging result.

To estimate the pulse duration $\hat{\tau}$ (used in DAS-E2 and RCB-E2), we select several typical signals (with clear peaks) and take the average of their pulse durations. In practice, the acoustic pulse duration is determined by the probing pulse duration, size and shape of the tumor, as well as the transducer response.

Fig. 4 shows the images for the simulated breast model with one 2-mm diameter tumor formed using ARMOR and DAS. The tumor response is weak for such a small tumor. In these images, we use $\varepsilon=0.1 \mathrm{M}$ and the searching range $[-14,14]$. Fig. 4(a) corresponds to DAS-C, where the tumor is buried by interference and noise. In Fig. 4(b), DAS-E1 fails to detect the tumor. In Fig. 4(c), for DAS-E2, a shadow of the tumor can be seen. In Fig. 4(d), for RCB-E2, most of the clutters are cleared up but a strong clutter shows up near the chest wall. Fig. 4(e)4(h) shows the results of peak searching; none of them have false tumors, which may be attributed to proper corrections of phase aberrations. Images produced by ARMOR-P in Fig. 4(f) 


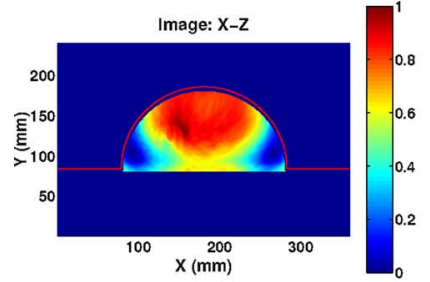

(a)

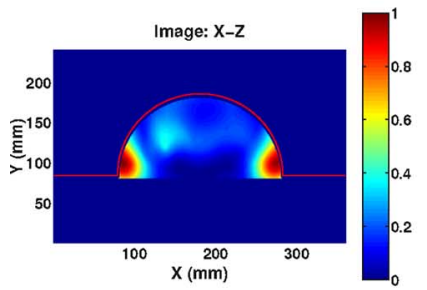

(c)

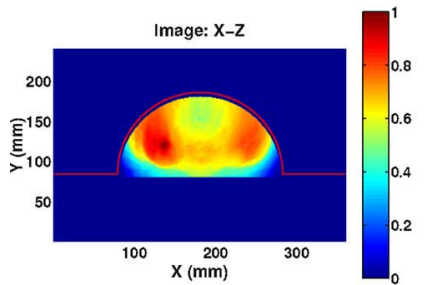

(e)

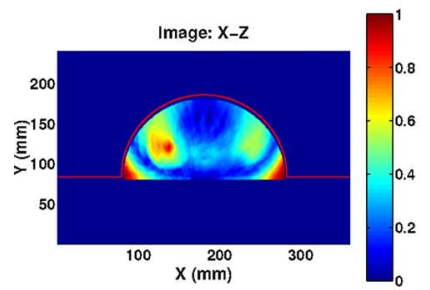

(g)

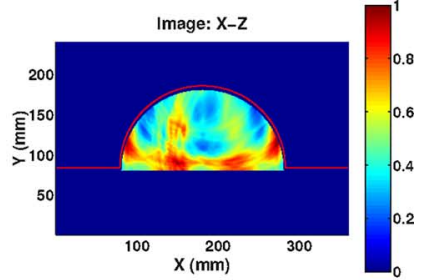

(b)

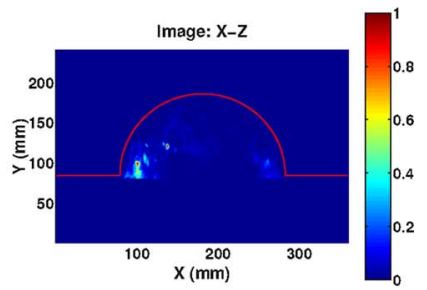

(d)

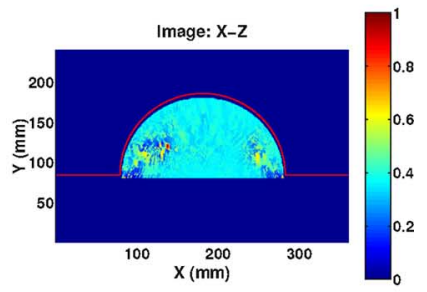

(f)

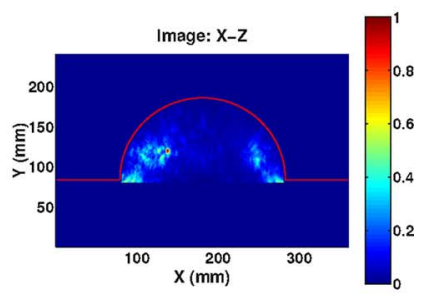

(h)

Fig. 4. Reconstructed images based on the 2-D simulated breast model with one 2-mm-diameter tumor. (a) DAS-C. (b) DAS-E1. (c) DAS-E2. (d) RCBE2, with $\varepsilon=0.1 M$. (e) DAS-P. (f) ARMOR-P, with $\varepsilon=0.1 M$. (g) DAS-PP. (h) ARMOR-PP, with $\varepsilon=0.1 M$.

and by ARMOR-PP in Fig. 4(h) have lower sidelobe levels and higher resolutions, and the latter has a higher contrast than the former, due to the latter using the peak-to-peak difference as the intensity measure.

Fig. 5 shows the imaging results for the one large tumor ( $1 \mathrm{~cm}$ diameter) case. Here, we set $\varepsilon=0.1 M$ and the searching range $[-20,20]$. (Note that different tumor sizes and locations will result in different sound speed variations in the breast model.) The white circle in the image corresponds to the actual contour of the tumor. Although all the methods can detect the tumor, only ARMOR can be used to form an image of the tumor with the best agreement with the actual tumor size and location.

By plotting a map (maps are not shown here due to limited space) of the values of $\mu$ used in ARMOR, for each focal point, we find that at the tumor locations, $\mu$ usually takes smaller values than that at other locations.

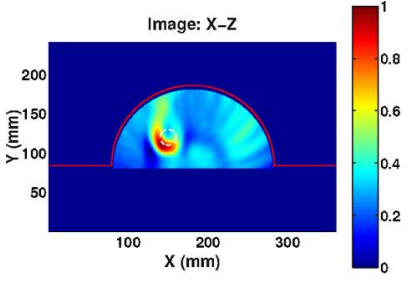

(a)

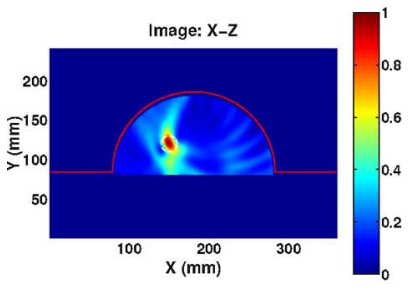

(c)

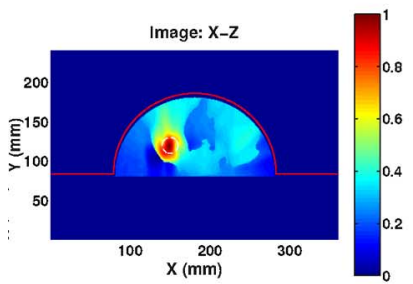

(e)

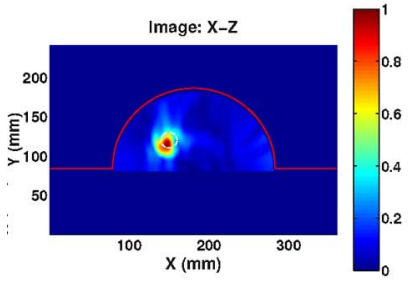

(g)

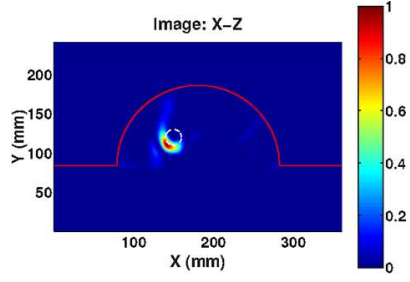

(b)

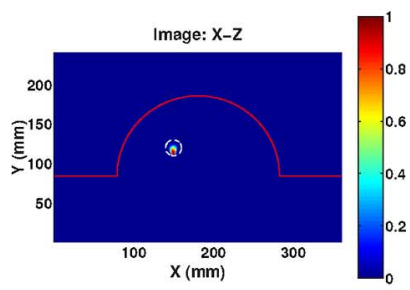

(d)

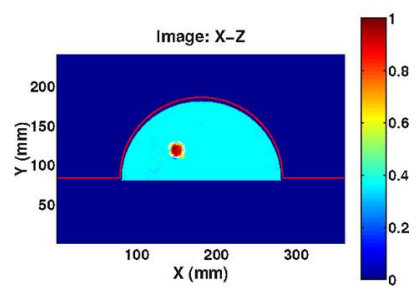

(f)

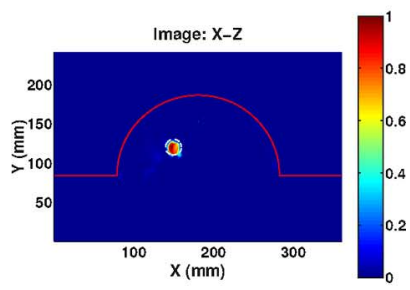

(h)
Fig. 5. Reconstructed images based on the 2-D simulated breast model with one large tumor ( $1 \mathrm{~cm}$ in diameter). The white circle in the image corresponds to the actual shape of the tumor. (a) DAS-C. (b) DAS-E1. (c) DAS-E2. (d) RCBE2, with $\varepsilon=0.1 M$. (e) DAS-P. (f) ARMOR-P, with $\varepsilon=0.1 M$. (g) DAS-PP. (h) ARMOR-PP, with $\varepsilon=0.1 M$.

\section{B. Experimental Results}

We have also tested ARMOR and DAS on two sets of TAT experimental data from mastectomy specimens [4] obtained by the Optical Imaging Laboratory at the Texas A\&M University.

The two data sets were acquired from mastectomy specimens using a TAT system. Microwave sources were used to heat the specimens transiently. In the experiment, the breast specimen was formed to a cylindrical shape inside a plastic bowl. The bowl was immersed in ultrasound coupling medium in a container. For breast specimen I, the acoustic signals were recorded at 240 equally spaced scanning stops on a circular track of radius $12.9 \mathrm{~cm}$. The thickness of this specimen was about $4 \mathrm{~cm}$ in a round plastic bowl of $17 \mathrm{~cm}$ in diameter. This lesion was diagnosed as an invasive metaplastic carcinoma with chondroid and squamous metaplasia. The size of the tumor was measured to be $35 \mathrm{~mm}$ in diameter by TAT, and $36 \mathrm{~mm}$ in diameter by radiography (see [4] for details). For breast specimen II, the scanning radius was $9.7 \mathrm{~cm}$, with 160 scanning stops. This specimen was 


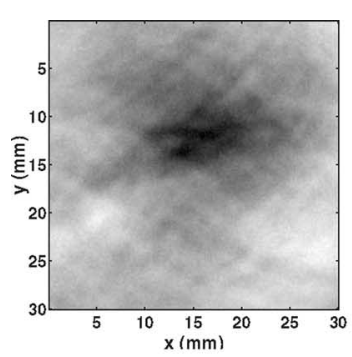

(a)

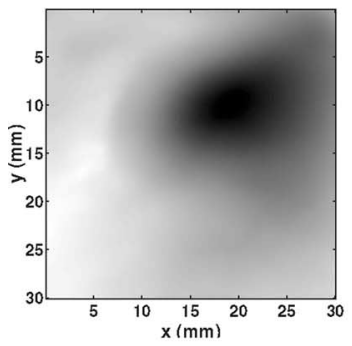

(d)

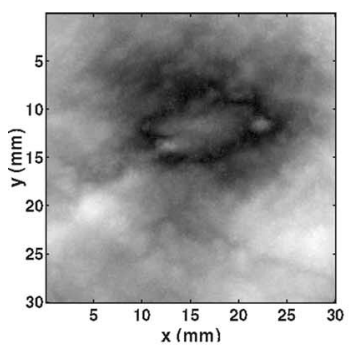

(g)

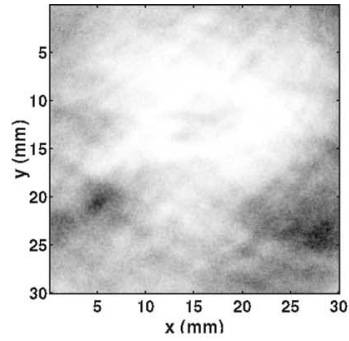

(b)

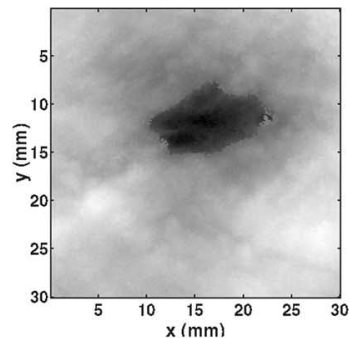

(e)

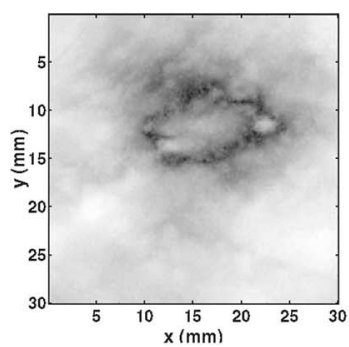

(h)

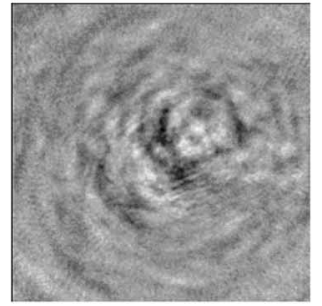

(j)

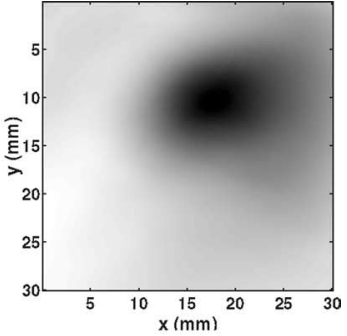

(c)

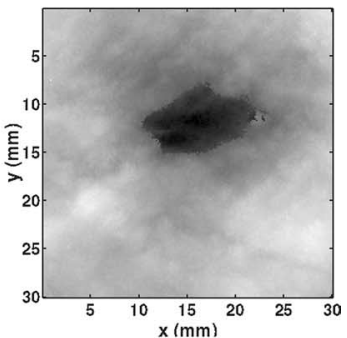

(f)

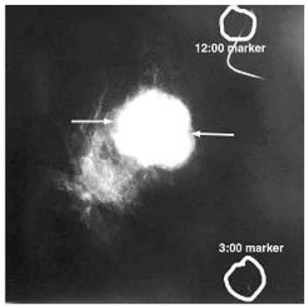

(i) $\varepsilon=0.5 M$. (g) DAS-PP. (h) ARMOR-PP, with $\varepsilon=0.5 M$. (i) X-ray image. (j) Inverse solution.

$9 \mathrm{~cm}$ thick in a round plastic bowl of $11 \mathrm{~cm}$ in diameter. The lesion in the specimen was diagnosed as infiltrating lobular carcinoma; the size of the tumor was about $20 \mathrm{~mm} \times 12 \mathrm{~mm}$ on TAT image, and about $26 \mathrm{~mm} \times 15 \mathrm{~mm}$ on the radiography (see [4] for more details).

First, we study the delay difference for both the breast specimens to get a qualitative guide for choosing the searching range in Step II of ARMOR. The results are shown in Fig. 3(c) and 3(d), respectively. Note that breast specimen II has a larger variance in delay differences than breast specimen I. In Fig. 3(c), $70 \%$ of the delay differences are roughly between -23 to 23 samples, whereas in Fig. 3(d), 70\% of the delay differences are between -40 and 40 samples. Therefore we should set a larger searching range for breast specimen II than for breast specimen I.
Fig. 6 shows the reconstructed images for breast specimen I. In the following images, the searching range was set to $[-3,3]$ after adjustment, and $\varepsilon=0.5 \mathrm{M}$ for all the RCBs used herein. In Fig. 6(a), for DAS-C, the dark region shows a blurred object corresponding to the breast tumor. In Fig. 6(b), for DAS-E1, the light region shows a vague boundary of the tumor. Fig. 6(c), for DAS-E2, and 6(d), for RCB-E2, have similar performances. In Fig. 6(e), for DAS-P, and 6(f), for ARMOR-P, a dark region with a clear cut has a good correspondence with the location and shape of the tumor in the radiograph [4]. In Fig. 6(g), for DAS-PP, and 6(h), for ARMOR-PP, not only a clear image of the tumor is obtained, but also the detailed boundary is revealed. For comparison, the images from X-ray mammography, considered the "gold standard" of breast imaging, and the exact inverse solution of TAT (see [4] for more details) are shown in Fig. 6(i) 


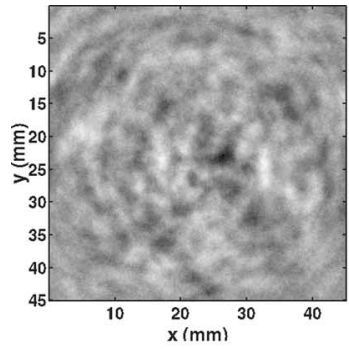

(a)

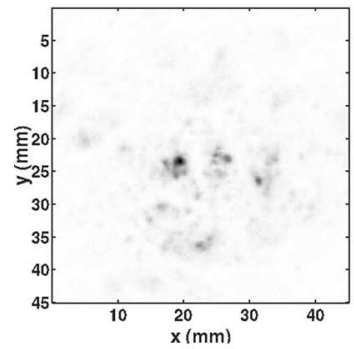

(d)

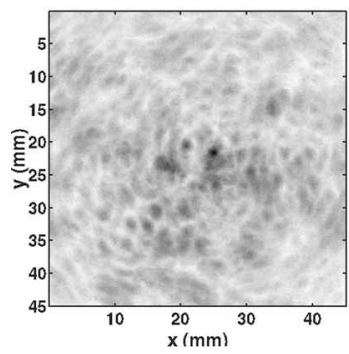

(g)

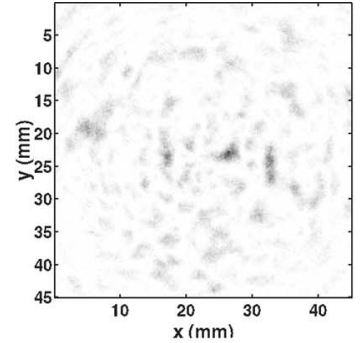

(b)

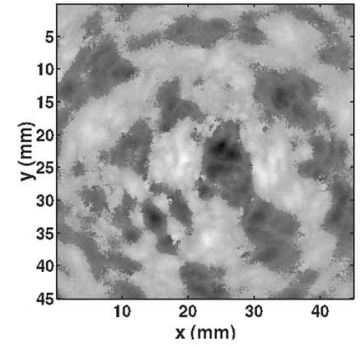

(e)

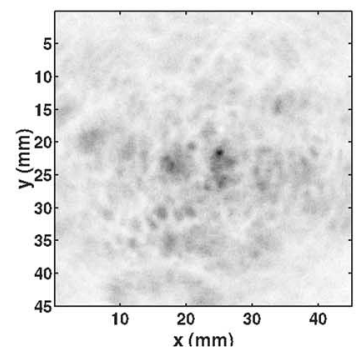

(h)

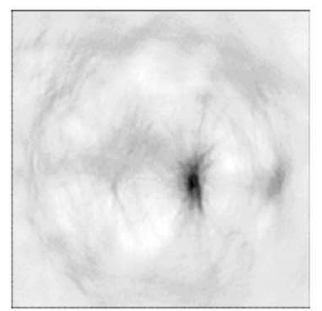

(j)

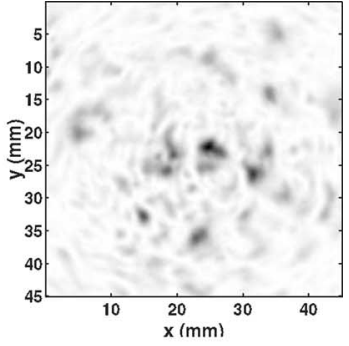

(c)

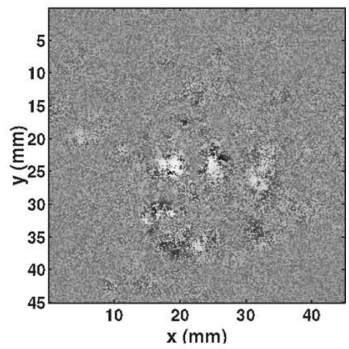

(f)

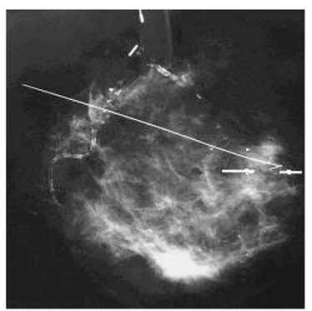

(i)

Fig. 7. Reconstructed images for breast specimen II. (a) DAS-C. (b) DAS-E1. (c) DAS-E2. (d) RCB-E2, with $\varepsilon=0.5 M$. (e) DAS-P. (f) ARMOR-P, with $\varepsilon=0.5 M$. (g) DAS-PP. (h) ARMOR-PP, with $\varepsilon=0.5 M$. (i) X-ray image. (j) Inverse solution.

and 6(j), respectively. We give Fig. 6 and the following Fig. 7 in gray scale to have a better comparison with the X-ray images.

Fig. 7 shows the reconstructed images for breast specimen II. The tumor size here is smaller, and a high level of interference and noise is present in the recorded data. The searching interval is eventually adjusted to $[-120,120]$ and RCB parameter $\varepsilon=0.5 M$. In Fig. 7(a), for DAS-C, the true tumor is barely identifiable from the surrounding clutters. The DAS-E1 shown in Fig. 7(b) and the DAS-E2 shown in Fig. 7(c) provide higher imaging contrast than DAS-C but show strong clutter. In Fig. 7(d), for RCB-E2, a false tumor shows up, which demonstrates the need for robustness in the presence of relatively strong phase distortion. DAS-P is shown in Fig. 7(e) and ARMOR-P is shown in Fig. 7(f). DAS-PP and ARMOR-PP produce the best images in Figs. 7(g) and 7(h), respectively, with the location and shape of the tumor consistent with the radiograph in Fig. 7(i) [4]. If we define the signal-to-background ratio (SBR) (i.e., squaring the pixel values of the image, the ratio of the maximum to the total sum of the squared values) as an image quality measurement metric, ARMOR-PP has an SBR twice that of DAS-PP, which means a $3 \mathrm{~dB}$ gain for ARMOR-PP. For comparison, the image formed by the exact inverse solution of TAT (see [4] for more details) is shown in Fig. 7(j).

The effects of the uncertainty parameter $\varepsilon$ in ARMOR is studied in our next example. We vary $\varepsilon$ of RCB used in ARMOR. The imaging results for breast specimen I, shown in Fig. 8, are consistent with our previous analysis: when $\varepsilon$ is large, the performance of RCB, in Fig. 8(a), is close to that of DAS in Fig. 6(g). When the parameter $\varepsilon$ is small, as shown in Fig. 8(c), the resolution is improved at the cost of robustness. 


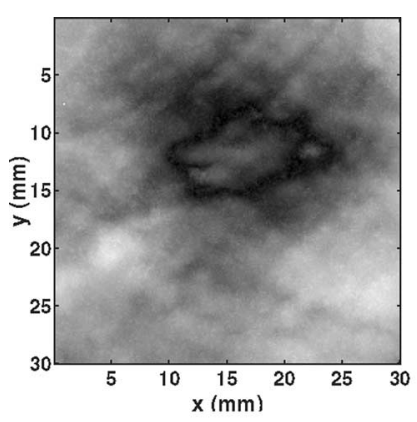

(a)

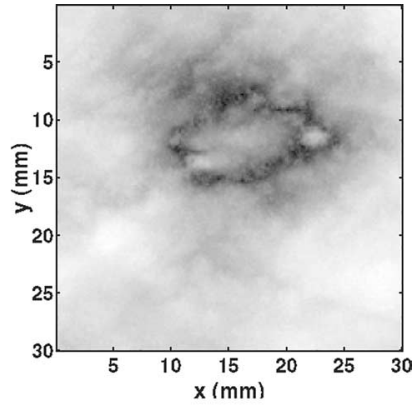

(b)

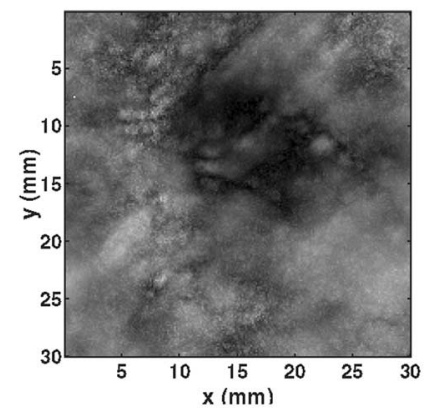

(c)

Fig. 8. Effects of uncertainty parameter $\varepsilon$ on ARMOR-PP, with a searching range $[-3,3]$. (a) $\varepsilon=0.7 M$. (b) $\varepsilon=0.5 M$. (c) $\varepsilon=0.3 M$.

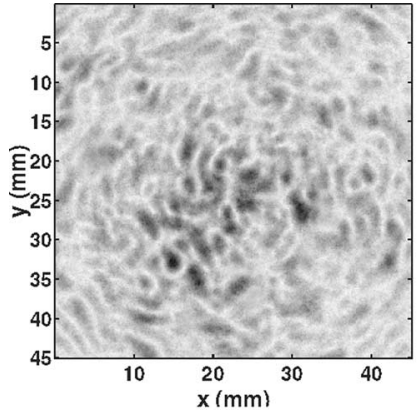

(a)

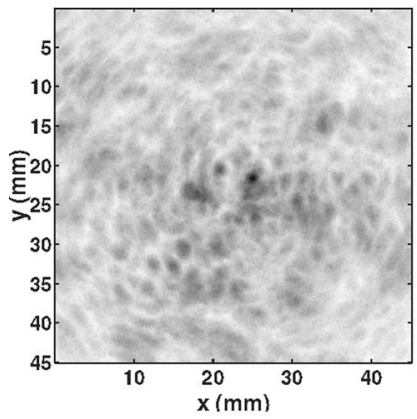

(c)

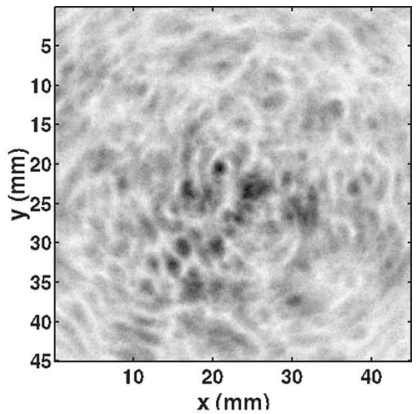

(b)

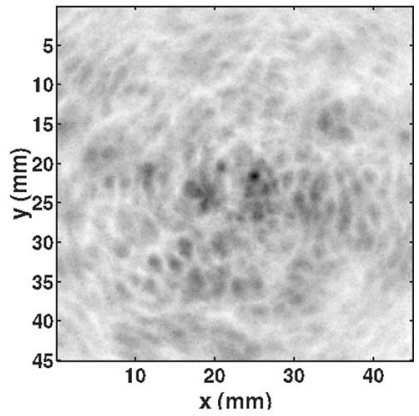

(d)
Fig. 9. Effects of the searching range on the DAS-PP images. (a) Searching range $[-20,20]$. (b) Searching range: $[-40,40]$. (c) Searching range $[-60,60]$. (d) Searching range: $[-80,80]$.

In our last example, the effect of the searching-range width on the imaging quality is considered. We use DAS-PP as an example since it shows more dependence on the searching range. The conclusion drawn for DAS applies to ARMOR. A symmetric searching range centered around the calculated arrival time is used. From the discussions in Section IV, we know that there is a tradeoff in choosing the searching range. Clearly, when the searching range is too small, such as in Fig. 9(a), we miss the true peaks. With an increase in the searching range, the image quality becomes gradually better, as shown in Fig. 9(b) and 9(c). However, when the searching range passes a certain threshold, with too much interference coming into the searching range, the image quality degrades because of increased clutters, as shown in Fig. 9(d).

From our numerical examples, we conclude that ARMOR has higher resolution and better interference rejection capability and more robustness against wavefront distortion than DAS. Also, we find that the amplitude-based measures reveal more details of the tumor in the reconstructed images than their energy-based counterparts. The energy-based measures are not sensitive to phase distortions; however, they tend to blur the reconstructed images, causing loss of details with a low-pass filtering-like effect.

\section{CONCLUSION}

ARMOR has been proposed for thermoacoustic tomography. ARMOR is robust to the amplitude and phase distortions in the recorded signals caused by the acoustic heterogeneity of biological tissues. ARMOR consists of three steps: in the first step, ARMOR uses the data-adaptive robust Capon beamforming ( $\mathrm{RCB}$ ) for waveform estimation; in the second step of ARMOR, a simple, yet effective, peak searching method is used to mitigate the phase distortion in the estimated waveform; in the third step, the response intensity is calculated for the focal point using various approaches, among which the peak-to-peak difference measure further enhances the image contrast. Examples based on a numerically simulated 2-D breast model and two sets of experimentally measured data from human mastectomy specimens demonstrate the excellent performance of ARMOR: high-resolution, low sidelobe level, and much improved interference suppression capability.

\section{APPENDIX}

\section{THERMAl ACOUSTIC Simulations}

We consider the microwave-induced thermal acoustic simulation in two steps. In the first step, the electromagnetic field inside the breast model is simulated and the specific absorption rate (SAR) distribution is calculated based on the simulated electromagnetic field. The second step is for the acoustic wave simulation, where the SAR distribution obtained in the first step is used as the acoustic pressure source through the thermal expansion coefficient. In both steps, the finite-difference time-domain (FDTD) method [28] is used for the simulation examples.

The 2-D electromagnetic breast model used is as shown in Fig. 2(a). A narrow electromagnetic pulse is used to irradiate the breast from the top of the model. The electromagnetic field is simulated using the FDTD method. The grid-cell size used by FDTD is $0.5 \mathrm{~mm} \times 0.5 \mathrm{~mm}$ and the computational region is terminated by perfectly matched layer (PML) absorbing boundary conditions [29]. 
TABLE II

Nominal DiElectric PROPERTIES OF BREAST TiSSuES [24]

\begin{tabular}{|l|c|c|}
\hline \multirow{2}{*}{ Tissues } & \multicolumn{2}{|c|}{ Dielectric Properties } \\
\cline { 2 - 3 } & Permittivity (F/m) & Conductivity (S/m) \\
\hline Immersion Liquid & 9 & 0 \\
\hline Chest Wall & 50 & 7 \\
\hline Skin & 36 & 4 \\
\hline Fatty Breast Tissue & 9 & 0.4 \\
\hline Nipple & 45 & 5 \\
\hline Glandular Tissue & $11-15$ & $0.4-0.5$ \\
\hline Tumor & 50 & 4 \\
\hline
\end{tabular}

TABLE III

ACOUSTIC PARAMETERS FOR BIOLOGICAL TISSUES

\begin{tabular}{|l|c|c|c|c|c|}
\hline Tissue & $\rho\left(\mathrm{kg} / \mathrm{m}^{3}\right)$ & $c(\mathrm{~m} / \mathrm{s})$ & $\alpha^{*}(\mathrm{~dB} / \mathrm{cm})$ & $\beta\left(1 /{ }^{\circ} \mathrm{C}\right)$ & $\left.C_{p}\left(\mathrm{~J} /{ }^{\circ} \mathrm{C} \cdot \mathrm{kg}\right)\right)$ \\
\hline Breast & 1020 & 1510 & $0.75 f^{1.5}$ & $3 \mathrm{E}-4$ & 3550 \\
\hline Skin & 1100 & 1537 & 3.5 & $3 \mathrm{E}-4$ & 3500 \\
\hline Muscle & 1041 & 1580 & $0.57 f$ & $3 \mathrm{E}-4$ & 3510 \\
\hline Tumor & 1041 & 1580 & $0.57 f$ & $3 \mathrm{E}-4$ & 3510 \\
\hline
\end{tabular}

$* f$ is the acoustic frequency, and the unit is in megahertz.

The SAR distribution is given as [30]

$$
\operatorname{SAR}(\mathbf{r})=\frac{\sigma(\mathbf{r}) E^{2}(\mathbf{r})}{2 \rho(\mathbf{r})}
$$

where $\sigma(\mathbf{r})$ is the conductivity of the biological tissues at location $\mathbf{r}, E(\mathbf{r})$ is the electric field at location $\mathbf{r}$, and $\rho(\mathbf{r})$ is the mass density of the biological tissues at location $\mathbf{r}$.

In the microwave-induced TAI system, the microwave energy is small, and as a result, the acoustic pressure field induced by the microwave is also small. So, the nonlinear acoustic effect does not need to be considered in the TAI system. The two basic linear acoustic wave generation equations are [9]

$$
\rho \frac{\partial}{\partial t} \mathbf{u}(\mathbf{r}, t)=-\nabla p(\mathbf{r}, t)
$$

and

$$
\nabla \cdot \mathbf{u}(\mathbf{r}, t)=-\frac{1}{\rho c^{2}} \frac{\partial}{\partial t} p(\mathbf{r}, t)+\alpha p(\mathbf{r}, t)+\beta \frac{\partial}{\partial t} T(\mathbf{r}, t)
$$

where $\mathbf{u}(\mathbf{r}, t)$ is the acoustic velocity vector, $p(\mathbf{r}, t)$ is the acoustic pressure field, $\rho$ is the mass density, $\alpha$ is the attenuation coefficient, $\beta$ is the thermal expansion coefficient, and $T(\mathbf{r}, t)$ is the temperature. The values for these acoustic properties for different breast tissues are listed in Table III [25].

Because the duration of the microwave pulse is much shorter than the thermal diffusion time, thermal diffusion can be neglected [9], and the thermal equation is

$$
C_{p} \frac{\partial}{\partial t} T(\mathbf{r}, t)=\operatorname{SAR}(\mathbf{r}, t)
$$

where $C_{p}$ is the specific heat. Substituting (29) into (28) gives

$$
\nabla \cdot \mathbf{u}(\mathbf{r}, t)=-\frac{1}{\rho c^{2}} \frac{\partial}{\partial t} p(\mathbf{r}, t)+\alpha p(\mathbf{r}, t)+\frac{\beta}{C_{p}} \operatorname{SAR}(\mathbf{r}, t) .
$$

FDTD is used again to compute the thermal acoustic wave based on (27) and (30).

The breast model for the acoustic simulation is shown in Fig. 2(b), which is constructed similarly to the model for electromagnetic simulation. An acoustic sensor array deployed uni- formly around the breast model is used to record the thermal acoustic signals. The grid-cell size used by the acoustic FDTD is $0.1 \mathrm{~mm} \times 0.1 \mathrm{~mm}$ and the computational region is terminated by PML-absorbing boundary conditions. Note that the size of the FDTD cell for the acoustic simulation is much finer than that of the FDTD cell for the electromagnetic simulation because the wavelength of an acoustic wave is much smaller than that of a microwave. The SAR distribution data is interpolated to achieve a desired grid resolution for the acoustic breast model.

\section{REFERENCES}

[1] R. G. Olsen and J. C. Lin, "Acoustic imaging of a model of a human hand using pulsed microwave irradiation," Bioelectromagnetics, vol. 4, pp. 397-400, 1983.

[2] R. A. Kruger, P. Liu, Y. R. Fang, and C. R. Appledorn, "Photoacoustic ultrasound (PAUS)—reconstruction tomography," Med. Phys., vol. 22, pp. 1605-1609, Oct. 1995.

[3] R. A. Kruger, K. D. Miller, H. E. Reynolds, W. L. Kiser, D. R. Reinecke and G. A. Kruger, "Breast cancer in vivo: Contrast enhancement with thermoacoustic CT at $434 \mathrm{MHz}$-feasibility study," Radiology, vol. 216, pp. 279-283, 2000.

[4] G. Ku, B. D. Fornage, X. Jin, M. Xu, K. K. Hunt, and L. V. Wang, "Thermoacoustic and photoacoustic tomography of thick biological tissues toward breast imaging," Technol. Cancer Res. Treat., vol. 4, pp. 1-7, Oct. 2005.

[5] S. S. Chaudhary, R. K. Mishra, A. Swarup, and J. M. Thomas, "Dielectric properties of normal and malignant human breast tissues at radiowave and microwave frequencies," Indian J. Biochem. Biophys., vol. 21, pp. 76-79, Feb. 1984.

[6] M. A. Anastasio, J. Zhang, X. Pan, Y. Zou, G. Ku, and L. V. Wang, "Halftime image reconstruction in thermoacoustic tomography," IEEE Trans. Med. Imag., vol. 24, no. 2, pp. 199-210, Feb. 2005.

[7] Y. Xu, D. Feng, and L. V. Wang, "Exact frequency-domain reconstruction for thermoacoustic tomography: I. Planar geometry," IEEE Trans. Med. Imag., vol. 21, no. 7, pp. 823-828, Jul. 2002.

[8] Y. Xu, M. Xu, and L. V. Wang, "Exact frequency-domain reconstruction for thermoacoustic tomography: II. Cylindrical geometry," IEEE Trans. Med. Imag., vol. 21, no. 7, pp. 829-833, Jul. 2002.

[9] M. Xu and L. V. Wang, "Time-domain reconstruction for thermoacoustic tomography in a spherical geometry," IEEE Trans. Med. Imag., vol. 21, no. 7, pp. 814-822, Jul. 2002.

[10] M. Xu, Y. Xu, and L. V. Wang, "Time-domain reconstruction algorithms and numerical simulations for thermoacoustic tomography in various geometries," IEEE Trans. Biomed. Eng., vol. 50, no. 9, pp. 1086-1099, Sep. 2003.

[11] C. G. A. Hoelen and F. F. M. de Mul, "Image reconstruction for photoacoustic scanning of tissue structures," Appl. Opt., vol. 39, no. 31, pp. 5872-5883, 2000.

[12] D. Feng, Y. Xu, G. Ku, and L. V. Wang, "Microwave-induced thermoacoustic tomography: Reconstruction by synthetic aperture," Med. Phys., vol. 28, pp. 2427-2431, Dec. 2001.

[13] Y. V. Zhulina, "Optimal statistical approach to optoacoustic image reconstruction,” Appl. Opt., vol. 39, no. 32, pp. 5971-5977, 2000.

[14] G. Kossoff, E. K. Fry, and J. Jellins, "Average velocity of ultrasound in the human female breast," J. Acoust. Soc. Amer, vol. 53, no. 6, pp. 17301736, 1973.

[15] T. Szabo, Diagnostic Ultrasound Imaging: Inside Out. New York: Academic, Sep. 2004.

[16] Y. Xu and L. V. Wang, "Effects of acoustic heterogeneity in breast thermoacoustic tomography," IEEE. Trans. Ultrason., Ferroelect. Freq. Control, vol. 50, no. 9, pp. 1134-1146, Sep. 2003.

[17] D. Liu and R. C. Waag, "Time-shift compensation of ultrasonic pulse focus degradation using least-mean-square error estimates of arrival time," $J$. Acoust. Soc. Amer., vol. 95, pp. 542-555, Jan. 1994.

[18] Q. Zhu and B. D. Steinberg, "Deaberration of incoherent wavefront distortion: An approach toward inverse filtering," IEEE Trans. Ultrason. Ferroelect, Freq. Control, vol. 44, no. 3, pp. 575-589, May 1997.

[19] J. Li, P. Stoica, and Z. Wang, "On robust Capon beamforming and diagonal loading," IEEE Trans. Signal Process, vol. 51, no. 7, pp. 1702-1715, Jul. 2003. 
[20] M. Xu and L. V. Wang, "Pulsed-microwave-induced thermoacoustic tomography: Filtered backprojection in a circular measurement configuration," Med. Phys., vol. 29, no. 8, pp. 1661-1669, 2002.

[21] J. Li and P. Stoica, Eds., Robust Adaptive Beamforming. New York: Wiley, 2005.

[22] Q. Zhu and B. D. Steinberg, "Wavefront amplitude distribution in the female breast," J. Acoust. Soc. Amer., vol. 96, pp. 1-9, Jul. 1994.

[23] X. Li and S. C. Hagness, "A confocal microwave imaging algorithm for breast cancer detection," IEEE Microw. Wireless Compon. Lett., vol. 11, no. 3, pp. 130-132, Mar. 2001.

[24] B. Guo, Y. Wang, J. Li, P. Stoica, and R. Wu, "Microwave imaging via adaptive beamforming methods for breast cancer detection," J. Electromagn. Waves Appl., vol. 20, no. 1, pp. 53-63, 2006.

[25] E. J. Bond, X. Li, S. C. Hagness, and B. D. Van Veen, "Microwave imaging via space-time beamforming for early detection of breast cancer," IEEE Trans. Antennas Propag., vol. 51, no. 8, pp. 1690-1705, Aug. 2003.

[26] C. Gabriel, R. W. Lau, and S. Gabriel, "The dielectric properties of biological tissues: II. Measured in the frequency range $10 \mathrm{~Hz}$ to $20 \mathrm{GHz}$," Phys. Med. Biol., vol. 41, pp. 2251-2269, Nov. 1996.

[27] Y. Sumino and R. Waag, "Measurements of ultrasonic pulse arrival time differences produced by abdominal wall specimens," J. Acoust. Soc. Am., vol. 90, pp. 2924-2930, Dec. 1991.

[28] A. Taflove and S. C. Hagness, Computational Electrodynamics: The Finite-Difference Time-Domain Method, 3rd ed. Boston, MA: Artech House, Jul. 2005.

[29] S. D. Gedney, "An anisotropic perfectly matched layer-absorbing medium for the truncation of FDTD lattices," IEEE Trans. Antennas Propag., vol. 44, no. 12, pp. 1630-1639, Dec. 1996.

[30] P. Bernardi, M. Cavagnaro, S. Pisa, and E. Piuzzi, "SAR distribution and temperature increase in an anatomical model of the human eye exposed to the field radiated by the user antenna in a wireless LAN," IEEE Trans. Microw. Theory Tech., vol. 46, no. 12, pp. 2074-2082, Dec. 1998.

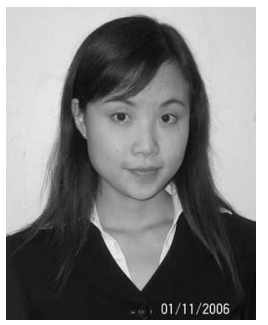

Yao Xie (S'04) received the B.Sc. degree from the University of Science and Technology of China (USTC), Hefei, China, in 2004, and the M.Sc. degree from the University of Florida, Gainesville, in 2006 , both in electrical engineering. She is currently working toward the Ph.D. degree at the Department of Electrical Engineering, Stanford University, Stanford, CA.

Her current research interests include optimization, information theory, and their applications in wireless communications, signal processing, and medical imaging.

Ms. Xie is a member of Tau Beta Pi and Etta Kappa Nu. She was the firstplace winner in the Student Best Paper Contest at the 2005 Annual Asilomar Conference on Signals, Systems, and Computers, for her work on breast cancer detection.

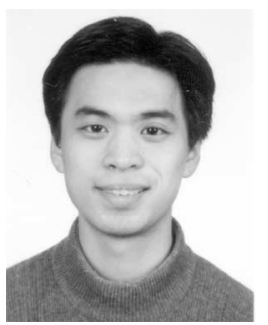

Bin Guo (S'05-M'07) received the B.E. and M.S degrees in electrical engineering from Xian Jiaotong University, Xian, China, in 1997 and 2000, respectively, and the Ph.D. degree from the Department of Electrical and Computer Engineering, University of Florida, Gainesville, in 2007.

Currently, he is a Research Associate in the Department of Electrical and computer Engineering, Duke University, Durham, NC. Prior to joining Duke University, he was a Research Assistant with the Department of Electrical and Computer Engineering, University of Florida, from 2003 to 2007, and was an Associate Research Scientist with the Temasek Laboratories, National University of Singapore, Singapore, from 2002 to 2003 . His current research interests include signal processing, microwave imaging, and computational electromagnetics.

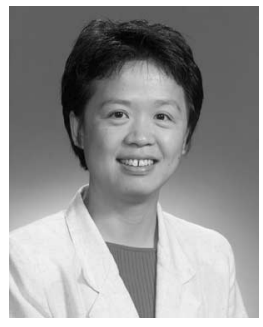

Jian Li (S'87-M'91-SM'97-F'05) received the M.Sc. and Ph.D. degrees in electrical engineering from Ohio State University, Columbus, in 1987 and 1991, respectively.

From April 1991 to June 1991, she was an Adjunct Assistant Professor with the Department of Electrical Engineering, Ohio State University. From July 1991 to June 1993, she was an Assistant Professor with the Department of Electrical Engineering, University of Kentucky, Lexington. Since August 1993, she has been with the Department of Electrical and Computer Engineering, University of Florida, Gainesville, where she is currently a Professor. Her current research interests include spectral estimation, statistical and array signal processing, and their applications.

Prof. Li is a Fellow of the Institution of Engineering and Technology (IET). She is a member of Sigma Xi and Phi Kappa Phi. She received the 1994 National Science Foundation Young Investigator Award and the 1996 Office of Naval Research Young Investigator Award. She is presently a member of two of the IEEE Signal Processing Society technical committees: the Signal Processing Theory and Methods (SPTM) Technical Committee and the Sensor Array and Multichannel (SAM) Technical Committee. She was an Executive Committee Member of the 2002 International Conference on Acoustics, Speech, and Signal Processing, Orlando, FL, in May 2002. She was an Associate Editor of the IEEE TRANSACTIONS ON SIGNAL PROCESSING from 1999 to 2005, an Associate Editor of the IEEE SignAL PROCESSING MAGAZINE from 2003 to 2005, and a member of the Editorial Board of Signal Processing, a publication of the European Association for Signal Processing (EURASIP), from 2005 to 2007. She has been a Member of the Editorial Board of Digital Signal Processing - $A$ Review Journal, a publication of Elsevier, since 2006. She is a coauthor of the papers that have received the First- and Second-Place Best Student Paper Award, respectively, at the 2005 and 2007 Annual Asilomar Conferences on Signals, Systems, and Computers in Pacific Grove, CA. She is also a coauthor of the paper that has received the M. Barry Carlton Award for the best paper published in IEEE TRANSACTIONS ON AEROSPACE AND ELECTRONIC SySTEMS in 2005.

Geng Ku received the B.S. and M.S. degrees from Huazhong University of Science and Technology, Wuhan, China, and the Ph.D. degree from the Department of Biomedical Engineering, Texas A\&M University, College Station.

$\mathrm{He}$ is a Research Faculty Member in the Department of Biomedical Engineering, Washington University at St. Louis, St. Louis. His current research interests include laser-based photoacoustic tomography and microwave-based thermoacoustic tomography.

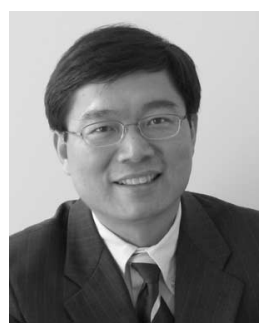

Lihong V. Wang (M'96-SM'00-F'06) received the $\mathrm{Ph} . \mathrm{D}$. degree from Rice University, Houston, TX, under the tutelage of Dr. Robert Curl, Dr. Richard Smalley, and Dr. Frank Tittel.

He holds the Gene K. Beare Distinguished Professorship in the Department of Biomedical Engineering, Washington University at St. Louis, St. Louis, and directs the Optical Imaging Laboratory. He invented or discovered frequency-swept ultrasoundmodulated optical tomography, dark-field confocal photoacoustic microscopy, photoacoustic Doppler sensing, photoacoustic reporter gene imaging, focused scanning microwaveinduced thermoacoustic tomography, exact reconstruction algorithms for photoacoustic or thermoacoustic tomography, sonoluminescence tomography, Mueller-matrix optical coherence tomography, and oblique-incidence reflectometry. His Monte Carlo model of photon transport in scattering media is used worldwide as a standard tool. He authored more than 137 peer-reviewed journal articles, and one of the first textbooks in biophotonics entitled Biomedical Optics Principles and Imaging (Wiley, 2007).

Prof. Wang is a Fellow of the American Institute for Medical and Biomedical Engineering (AIMBE), The Optical Society of America (OSA), and The International Society for Optical Engineering (SPIE). He serves on the editorial boards of the Journal of Biomedical Optics and Applied Optics. He is the Chair of the International Biomedical Optics Society. He was the recipient of the National Institutes of Health (NIH) FIRST award, the National Science Foundation (NSF) CAREER award, and the Outstanding Young Scientist Award sponsored by Johnson \& Johnson Medical and the Houston Society for Engineering in Medicine and Biology. 